\title{
Indian Sign Language Numeral Recognition - An Image Processing Approach
}

\author{
Pooja Kiranalli \\ Student, \\ Walchand Institute of Technology, \\ Solapur, India
}

\author{
S. R. Gengaje, PhD \\ H.O.D. Electronics Department, \\ Walchand Institute of Technology, \\ Solapur, India
}

\begin{abstract}
In recent years, Sign language is an important research problem for communicating with hearing impaired people without the help of interpreter. Hand gesture is one of the methods used in sign language which is most commonly used by deaf and dumb people to communicate with each other or with normal people. The proposed algorithm aims at developing real time image processing based system for hand gesture recognition on personal computer with an USB web cam. This paper proposes a method to detect and recognize the static image of Indian Sign Language numbering system from zero to nine. The method is based on counting the open fingers in the static images. The proposed algorithm for gesture recognition is based on boundary tracing and finger tip detection and also deals with images of bare hands, which allows the signer to interact with the system in a natural way. The proposed algorithm is first detect and segments the hand region from the real time captured images. Then using the proposed methodology, it locate the fingers and classifies the gesture. Further the system convert Indian signs into text and then speech using an audio file stored on PC. The algorithm is size invariant but it is orientation dependent. The proposed system is implemented using OpenCV.
\end{abstract}

\section{General Terms}

Gesture Recognition, Active finger counting.

\section{Keywords}

Hand Gesture Recognition(HGR), Human Computer Interation(HCI), Region of Interest (ROI).

\section{INTRODUCTION}

In everyday life, communication plays a major role. The basic idea of any communication system is to provide a means of exchanging information between the different parties involved in the communication. The ability to speak and hear plays an important role in day-to-day human-human interaction. Different socially aided people communicate in different ways. People with speech and hearing impairment find it especially difficult to communicate. The sign language serves as a medium of communication for these people. This proposal aims at developing a system which enhance the quality of communication for these people.

The sign language is a set of Signs that is used by signing deaf community to communicate among themselves. Sign language is a collection of gestures, movements, posters and facial expression corresponding to letters and words in natural languages. Sign languages are not international language. Different countries have unique sign languages. Like India have its own sign language (ISL) and is not a visual form of English. [1] Sign language recognition system act as a interpreter between common people and deaf-dumb people. It captures the gesture shown by the signer and convert it into known language. The term, Hand Gesture Recognition is collectively referred as, the process of tracking human gesture and then interpreting them in the form of meaningful commands.

Vision based hand gesture recognition is classified into two categories as static and dynamic gesture[3]. Static hand gestures are the orientation and position of hand in the space for fixed amount of time without any movement whereas dynamic hand gestures includes gestures with movement of hand like waving of hand or movements of fingers. The proposed algorithm is applicable only for static images. This paper propose an eccentric approach for hand gesture recognition which does not use the database of images for comparison, instead it simply capture the input image through a simple web cam then process it to count the number of active fingers and recognize the gesture by applying different logic for each gesture. This make it to recognize fast and hence can be used for real time application. The aim of the proposed system is to develop a PC based real time system for automatic recognition of static hand gesture of Indian (Devanagari) Sign Language numbers- zero to nine using image processing approach. Using our proposed algorithm, system recognized Indian Sign Language (ISL) number zero to nine as shown in figure 1
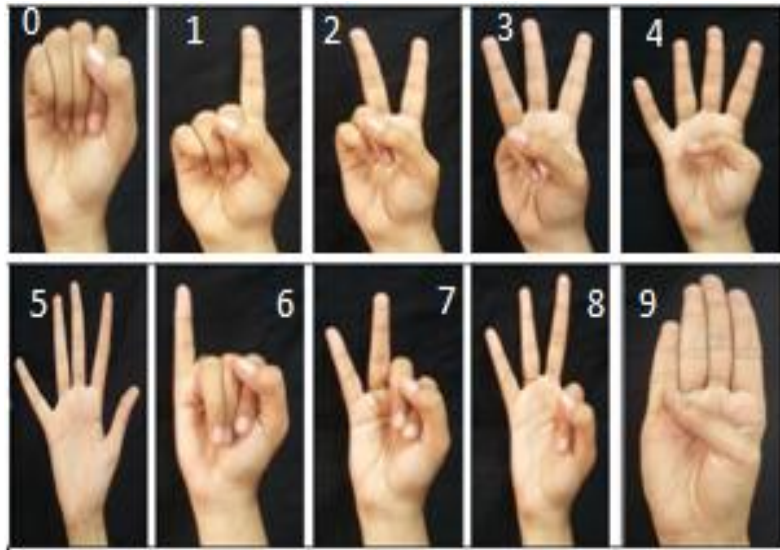

Figure 1. Indian Sign Language (ISL) numbering system from zero to nine

\section{RELATED WORK}

Digital image processing technology for HCI applications was inducted by the Space Foundation Space Technology Hall of Fame in 1994. There are few ways to perform hand gesture recognition. It is mainly classified into two categories. The first category contained heavy hardware parts such as glove based analysis. It uses sensors that are attached to a glove that translates finger flexions into electrical signal to determine the hand posture. Normally the sensors that are acoustic or 
magnetic sensor which embedded into the glove. Figure 2(a) shows the cyberglove II data gloves containing optical fibers as sensing units. Use of sensors and wires in dada gloves restricts the movements of user hand and hence limits its use in natural means of human computer interface.

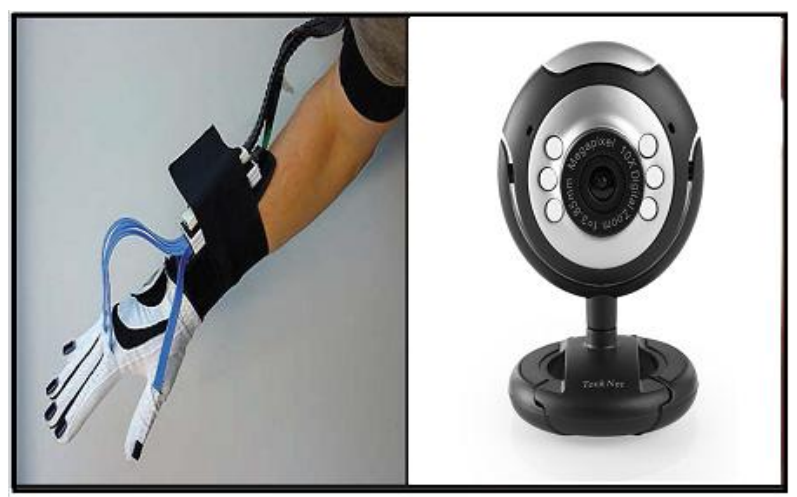

a) Cyberglove II

b) Simple web camera

Figure 2. Contact and Vision based devices used for hand gesture recognition

The second category is vision based analysis which is based on the way human beings realize information about their surroundings. It just need a camera, webcam, camcorder or anything that can capture image and able to interface with computer figure 2(b) shows the simple web camera that can be used to build any vision based system. The main advantage of this method is the degree of freedom for hand movement which is very essential in natural means of communication with machines. Visual based gesture recognition systems are further divided into two categories, gloves with visual markers and pure visual based gesture without any color gloves. The first one relies on using colored gloves or gloves with visual markers [2] that help to determine hand postures, But this do not provide the naturalness which required in human computer interaction system due to use of colored glove and markers. The other method relies on pure visual based gesture without any color gloves/ markers and uses images of bare hands to recognize gestures.

Most of the algorithms from literature uses conventional approach to build HCI system which uses feature extraction followed by feacher matching. Author in [4] proposed a system to recognize American Sing Language (ASL) gesture by using appearance based model. The proposed system can automatically translate static gesture of ASL alphabets using three different feature extraction methods and neural networks. V.G. Spitsyn and N.H. Phan in [5] proposed a novel algorithm based on Principal Component Analysis and wavelate transforms for hand as well as face recognition. Computer-aided interpreter for hearing and speech impaired is proposed in [6] which used using natural voice processing and digital image processing algorithms to converts the ASL gesture in text as well as in speech. K. Yeaol presented a static hand gesture recognition algorithm which uses k-mean based radial basis function neural network [7] in that pre-processing techniques are used for hand detection and tracking. Ruiduo yang [8] presented fast yet simple solution for addressing problems in static HGR. Author used Discrete Hidden Markov Models (DHMMs) that use features extracted from the hand contours. Daeho Lee and Youngtse Park in [9] proposed an algorithm to count number of open fingers. A convex-hull algorithm is first used to locate number of fingertips and for gesture classification.

\section{PROPOSED HAND GESTURE RECOGNITION SYSTEM}

Figure 3 shows block diagram of proposed system. The image of hand gesture is captured by a simple webcam. The captured image is in the RGB format but further algorithm deal with the gray scale images. Image segmentation is followed by some morphological operations and feature extraction for gesture recognition. Further by applying finger counting logic the number of active fingers are counted which further used for gesture classification.

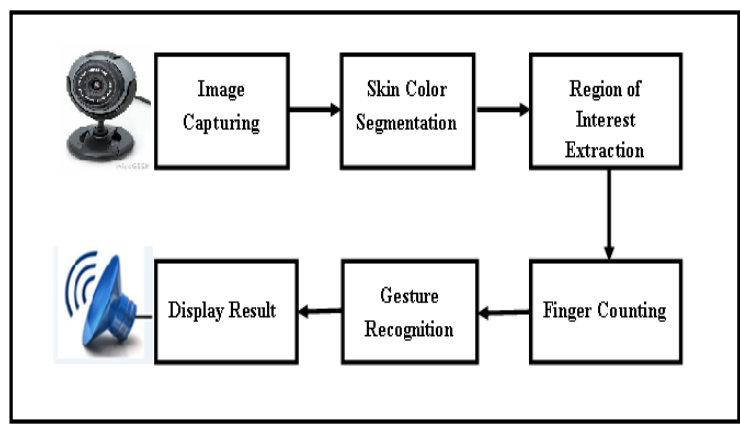

Figure.3. Block diagram of the proposed system

\subsection{Image capturing}

A 12 megapixel webcam is used to capture the input image which have a plain black background and with uniform illumination. The plain back background is used for simplicity and good segmentation. The captured image is of size $640 * 480$ and in the RGB format. In this paper, the proposed system is explained with the gesture of one, three and six as an example. Figure 4 shows captured images of one, three and six .
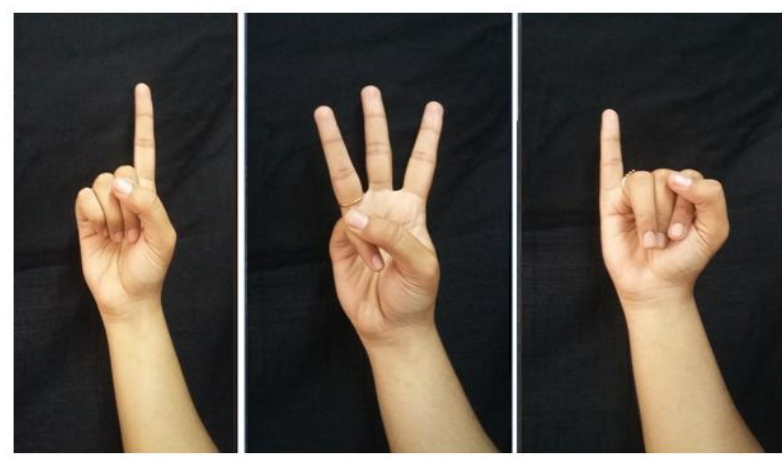

Figure 4. captured input image of ISL gesture-One, Three, Six

\subsection{Skin color segmentation}

To detect the hand region from background, a skin color detector has been implemented. A simple segmentation technique based on computing maximum and minimum skin probabilities of input RGB image is used. To avoid issues such as incomplete segmentation of finger regions and to preserve edges, morphological operations like erosion and dilation are used.

\subsection{Region of interest extraction}

The next step is to crop the image. This is done by finding the connected chain of pixels with value ' 1 ' using 8neigbourhood concept. Once all the chains are found, one with a longest value is selected and segregated from the image. This is the shape in the image which encloses the largest area that is our 'hand' region. Figure 5 shows the 
isolated hand region from the captured input RGB format image in a binary format image, where white pixel represent skin areas and black pixel represent non-skin areas.
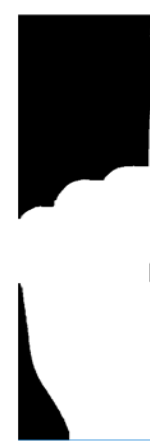
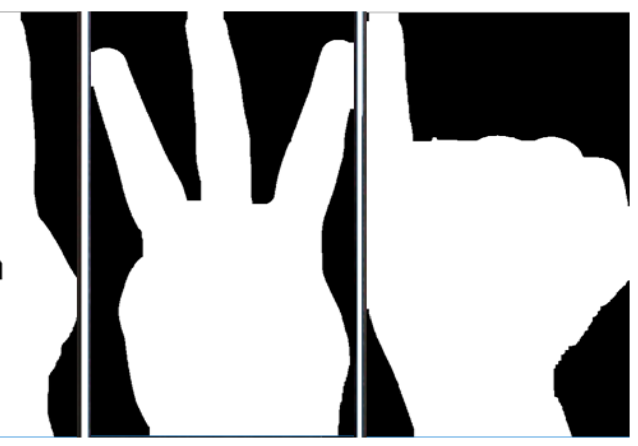

Figure 5. Extracted region of interest containing only hand region

\subsection{Finger counting}

After the segmentation and finding region of interest, the next step is to count the number of active fingers. There are two steps to count the number of active fingers. In first step only fingers of the hand are cropped from the cropped binary image. This is done by selecting y axis pixel and remaining pixels are masked. In proposed algorithm, this value is 300 . The second step is to count the contours from the image which got in first step. The count of contour is the required value of number of active finger. Fig 6 shows the finger count of one, three and six. For gesture zero, one, six and nine the count is 'one'. For gesture two and seven count is 'two'. For gesture three and eight count is 'three'. Gesture four and five directly counted as 'four' and 'five'. After that mean value is calculated from convex hull and defects point and from that standard deviation is calculated which is used to classify the gesture. Fig 7 shows the convex hull and defect points of gesture one, three and six. Red point shows the convex hull whereas green and blue point shows its defects point
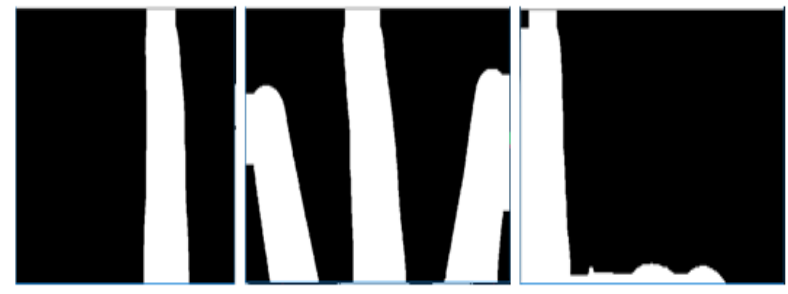

Figure 6. finger count of gesture one, three and six

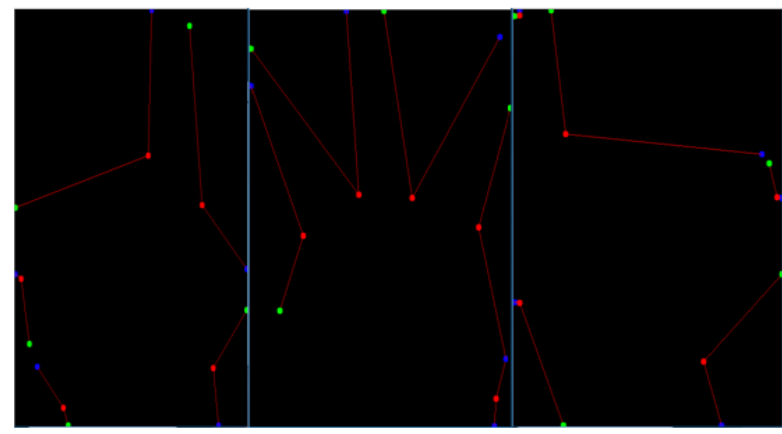

Figure 7. Convex hull and Defect point of gesture one, three and six

\subsection{Gesture recognition}

In this phase, each ISL gesture is classified based on its count value and by using few logical conditions. The gesture one to five are recognized correctly. The gesture four and five can be recognized directly but the classification is needed for the gesture of zero, one, two, three, six, seven, eight, nine. For gesture 0 detection convex hull and its defects are used. Using this points standard deviation is calculated. For gesture zero standard deviation is minimum. Gesture 1-3 detection is done by using simple finger counting logic. For gesture 5 detection convex hull points and its defects are used. Gesture 6-8 are recognized based on which quadrant it belongs to and finally for 9 area parameters are considered.

\subsection{Display result}

The recognized hand gestures are display in the form of number figure and audio of that same number. Figure 8 shows the result of given input gesture one, three and six.

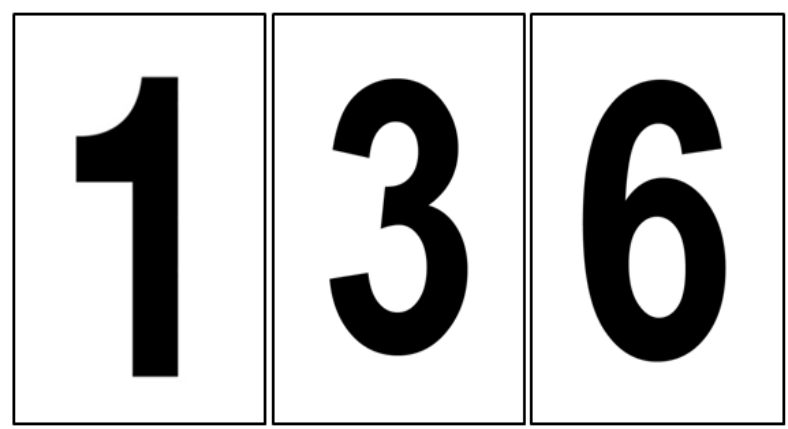

Figure 8. Recognized gesture as one, three and six

\section{EXPERIMENTAL RESULTS}

The proposed algorithm is implemented using OpenCV library and tested for each gesture. All the images are taken against back background under uniform lightening condition.

In the experiment, each gesture is checked for 15 times, so there are 150 samples of hand gestures are collected from 15 different individuals having different hand size. To classify the gesture mean and standard deviation is calculated from the convex hull and defects point. Table 1 gives the average of mean and standard deviation of 15 images of gesture zero to nine and figure 9 shows the graphical representation of the same.

Table 1. Average of Mean and Standard deviation

\begin{tabular}{|c|c|c|}
\hline $\begin{array}{c}\text { Input ISL } \\
\text { Gesture }\end{array}$ & $\begin{array}{c}\text { Average of } \\
\text { Mean }\end{array}$ & $\begin{array}{c}\text { Average of Standard } \\
\text { deviation }\end{array}$ \\
\hline Zero & 1500 & 1265 \\
\hline One & 8164 & 3799 \\
\hline Two & 14500 & 8071 \\
\hline Three & 1500 & 1265 \\
\hline Four & 20000 & 12300 \\
\hline Five & 19141 & 13904 \\
\hline Six & 3711 & 1311 \\
\hline Seven & 11300 & 4023 \\
\hline Eight & 1500 & 1265 \\
\hline Nine & 19141 & 13904 \\
\hline
\end{tabular}




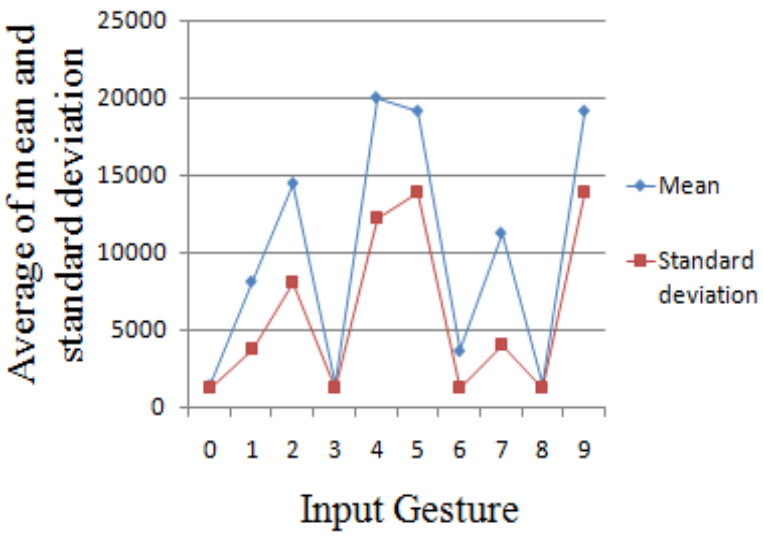

Figure 9. Graphical representation of Average of Mean and Standard deviation

The algorithm is applied on 15 images of each gesture as well as each gesture is checked for 15 times with different size of hand and lightening condition in real time. The recognition result and accuracy of each gesture is recorded as given in table 2. Figure 10 gives the graphical representation of the same. Blue bar shows the accuracy (in percentage) of gestures in offline mode whereas red bar shows the accuracy (in percentage) of gesture in real time i.e. online mode. Recognition accuracy is calculated using following equation.

Recognition accuracy $=\frac{\begin{array}{c}\text { Number of gesture correctly } \\ \text { recognised }\end{array}}{\text { Total number of input gesture }}$

Table 2. Experimental result of recognition ISL gesture

\begin{tabular}{|c|c|c|c|c|}
\hline $\begin{array}{c}\text { Input } \\
\text { ISL } \\
\text { Gesture }\end{array}$ & $\begin{array}{c}\text { Correctly } \\
\text { recognized } \\
\text { out of } 15 \\
\text { (offline) }\end{array}$ & $\begin{array}{c}\text { Accuracy } \\
(\%)\end{array}$ & $\begin{array}{c}\text { Correctly } \\
\text { recognized } \\
\text { out of } 15 \\
\text { (online) }\end{array}$ & $\begin{array}{c}\text { Accuracy } \\
(\%)\end{array}$ \\
\hline Zero & 14 & 93.33 & 15 & 100 \\
\hline One & 15 & 100 & 15 & 100 \\
\hline Two & 15 & 100 & 15 & 100 \\
\hline Three & 14 & 93.33 & 15 & 100 \\
\hline Four & 15 & 100 & 14 & 93.33 \\
\hline Five & 13 & 86 & 13 & 86 \\
\hline Six & 15 & 100 & 15 & 100 \\
\hline Seven & 15 & 100 & 15 & 100 \\
\hline Eight & 14 & 93.33 & 14 & 93.33 \\
\hline Nine & 13 & 86 & 13 & 86 \\
\hline
\end{tabular}

Thus the overall accuracy of the proposed system is $95.86 \%$ for offline mode and $95.19 \%$ for offline mode.

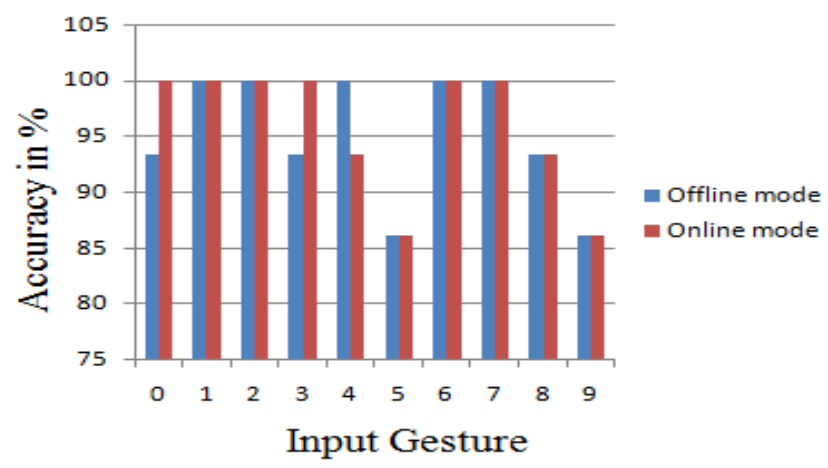

Figure 10. Experimental result of recognition ISL gesture

\section{CONCLUSION}

Using proposed methodology, zero to nine ISL sign numbers are recognized. The proposed system is tested in different lighting conditions with plain back background. In future, the algorithm can be improved so that gesture with non-uniform background can be recognized. The algorithm is size invariant but orientation dependent. In future, the algorithm may applicable for various orientations of hand and in varying light conditions by improving algorithm. The program is written in OpenCV using C++ language and tested in real time environment. The algorithm is simple and do not use any feature extraction/comparison hence it takes very less computational time which makes it use for developing real time HCI application.

\section{REFERENCES}

[1] V. Bhame, R. Sreemathy, H. Dhumal, "Vision Based Calculator for Speech and Hearing Impaired using Hand Gesture Recognition,"1nternational Journal of Engineering Research \& Technology (IJER1], Vol. 3, Issue 6, June - 2014.

[2] G.Fang, W.Gao, J. Ma, "Signer-independent sign language recognition based on SOFM/HMM", Fifth IEEE International Conference on Systems, Man, and Cybernetics, vol.2,pp.132-140,4-6 April 2012.

[3] V. Bhame, R. Sreemathy, H. Dhumal, "Vision Based Hand Gesture Recognition Using Eccentric Approach for Human Computer Interaction", 2014 International Conference on Advances in Computing. Communications and Informatics (ICACCI).

[4] V. Kulkarni and S. Lokhande, "Appearance Based Recognition of American Sign Language Using Gesture Segmentation", International Jouranal on Computer Science and Engineering (IJCSE), ISSN: 0975-3397, vol. 02, No, 03, pp.560-565, March 2010.

[5] Joon-Kee Cho, Dong Ryeol park and Yeon-Ho Kim, "A Method of Remote Control for Home Appliance Using Free Hand Gesture", IEEE International conference on Consumer Electronics, ISSN: 0673-3867, June 2012.

[6] Prashanth Suresh and Niraj Vasudevan, "Computer-aided interpreter for hearing and speech impaired", Fourth International Conference on Computational Intelligence, Communication Systems and Networks, June 2012.

[7] Kook-Yeol Yoo, "Robust Hand Segmentation and Tracking to Illumination Variation", in Proc. IEEE Internatioanl Conference on Consumer Electronics (ICCE), September 2014.

[8] Ruiduo Yang and Sudeep Sarkar," Gesture Recognition using Hidden Markov Models From Fragmented Observations",in Proc. IEEE Computer Society Conference on Computer Vision and Pattern Recognition (CVPR'06), June 2006.

[9] Daeho Lee and Youngtae Park,"Vision-Based Remote Control System by Motion Detection and Open Finger Counting", in Proc. IEEE Transactions on Consumer Electronics, Vol.55, No.4, November 2009. 